(2) Open Access Full Text Article

\title{
A study to assess COPD Symptom-based Management and to Optimise treatment Strategy in Japan (COSMOS-J) based on GOLD 20I I
}

This article was published in the following Dove Press journal: International Journal of Chronic Obstructive Pulmonary Disease 2 October 2013

Number of times this article has been viewed

\section{Tomoko Betsuyaku' \\ Motokazu Kato² \\ Keisaku Fujimoto 3 \\ Gerry Hagan ${ }^{4}$ \\ Akihiro Kobayashi ${ }^{5}$ \\ Hideki Hitosugi ${ }^{5}$ \\ Mark James 5 \\ Paul W Jones ${ }^{6}$}

'Division of Pulmonary Medicine, Department of Medicine, Keio University, Tokyo, Japan; ${ }^{2}$ Department of Respiratory Disease, Kishiwada City Hospital, Osaka, Japan; ${ }^{3}$ Department of Clinical Laboratory Sciences, Shinshu University, Nagano, Japan; ${ }^{4}$ Private Practice, Marbella, Spain; ${ }^{5}$ GlaxoSmithKline KK, Tokyo, Japan; ${ }^{6}$ Division of Clinical Science, St George's, University of London, London, UK
Correspondence: Paul W Jones Division of Clinical Science, St George's, University of London, Blackshaw Road, London, SWI7 0QT, UK

Email pjones@sgul.ac.uk
Background and objective: The Global initiative for chronic Obstructive Lung Disease (GOLD) Committee has proposed a chronic obstructive pulmonary disease (COPD) assessment framework focused on symptoms and on exacerbation risk. This study will evaluate a symptom and exacerbation risk-based treatment strategy based on GOLD in a real-world setting in Japan. Optimal management of COPD will be determined by assessing symptoms using the COPD Assessment Test (CAT) and by assessing the frequency of exacerbations.

Methods: This study (ClinicalTrials.gov identifier: NCT01762800) is a 24-week, multicenter, randomized, double-blind, double-dummy, parallel-group study. It aims to recruit 400 patients with moderate-to-severe COPD. Patients will be randomized to receive treatment with either salmeterol/fluticasone propionate (SFC) $50 / 250 \mu \mathrm{g}$ twice daily or with tiotropium bromide $18 \mu \mathrm{g}$ once daily. Optimal management of patients will be assessed at four-weekly intervals and, if patients remain symptomatic, as measured using the CAT, or experience an exacerbation, they have the option to step up to treatment with both drugs, ie, SFC twice daily and tiotropium once daily (TRIPLE therapy). The primary endpoint of the study will be the proportion of patients who are able to remain on the randomized therapy.

Results: No data are available. This paper summarizes the methodology of the study in advance of the study starting.

Conclusion: The results of this study will help physicians to understand whether TRIPLE therapy is more effective than either treatment strategy alone in controlling symptoms and exacerbations in patients with moderate-to-severe COPD. It will also help physicians to understand the GOLD recommendation work in Japan.

Keywords: COPD, GOLD, symptom, exacerbation risk, TRIPLE therapy

\section{Introduction}

Chronic obstructive pulmonary disease (COPD) is a common chronic inflammatory disease characterized by persistent airflow limitation, which is both preventable and treatable. ${ }^{1}$ The burden of COPD is considerable and increasing. ${ }^{2,3}$

The severity of COPD is influenced by exacerbations and comorbidities. ${ }^{4}$ Spirometry has remained the standard method for confirming a clinical diagnosis of COPD and for assessing COPD severity, ${ }^{5}$ but it is now accepted that forced expiratory volume in 1 second $\left(\mathrm{FEV}_{1}\right)$ is an inadequate marker of the severity of breathlessness, exercise limitation, and health status impairment, ${ }^{1,4}$ although it remains important in the confirmation of a clinical diagnosis of COPD.

The Global initiative for chronic Obstructive Lung Disease (GOLD) strategy document, updated in 2011 and 2013, has proposed a new, multidimensional approach 
to assess patients with COPD. It recommends that COPD management and treatment should consider both disease impact, determined by assessment of symptoms and activity limitation, and future risk of exacerbations, determined from airflow limitation or exacerbation history. ${ }^{5,6}$ This combined assessment of COPD results in the grouping of patients into one of four categories: (A) low risk, fewer symptoms; (B) low risk, more symptoms; (C) high risk, fewer symptoms; (D) high risk, more symptoms.

For assessing symptoms, GOLD advises utilizing either the COPD Assessment Test (CAT) or the modified Medical Research Council (mMRC) dyspnea scale. The CAT is a simple tool for quantifying the symptoms and impacts of $\mathrm{COPD}^{7,8}$ that has been shown to distinguish between different severities of COPD, is highly correlated with the St George's Respiratory Questionnaire (SGRQ) ${ }^{7}$ and has been validated in Japan. ${ }^{9}$

In Japan, the name recognition of COPD is low, and the majority of these patients have not been diagnosed or appropriately treated. ${ }^{10}$ The prevalence of COPD is also underestimated in Japan. ${ }^{11,12}$ These findings highlighted the urgent need for improvements in COPD diagnosis and management in general practice. Most diagnosed patients are treated with bronchodilators such as long-acting $\beta_{2}$ agonists (LABAs) or long-acting antimuscarinics (LAMAs) as monotherapy, and remain symptomatic. ${ }^{13}$ It has now been recognized that monotherapy may not enable many patients to achieve the goals of pharmacologic therapy for COPD, namely to control symptoms, improve health status, and reduce the frequency of exacerbations. ${ }^{1}$ Guidelines on COPD management recommend the combined use of long-acting bronchodilators and inhaled corticosteroids (ICS) to optimize outcomes, especially exacerbations, in patients with COPD that is inadequately controlled with monotherapy. ${ }^{1}$ It has been demonstrated that, in some patients, TRIPLE therapy, in which an anticholinergic is added to an ICS and LABA, has been associated with greater improvements in lung function and quality of life and with reduced rates of hospitalization compared with anticholinergic therapy alone. ${ }^{13-17}$

The aim of this innovative study is to assess the control of COPD using a symptom and exacerbation risk-based treatment strategy according to GOLD 2011. This study has been named the "COPD Symptom-based Management and to Optimise treatment Strategy in Japan" (COSMOS-J) and has been designed to reflect clinical practice in Japan. Unlike a "traditional" randomized controlled trial, treatment in this study is flexible as it tries to reflect clinical practice. This will demonstrate whether the treatment approach outlined in the GOLD strategy document can be used to improve the current management of COPD in Japan. New Japanese guidelines are also anticipated in 2013, which may improve clinical practice. Optimal management of COPD, determined by assessing symptoms using CAT and by assessing the frequency of exacerbations, will be assessed in patients who have been randomized to receive treatment with either salmeterol/fluticasone propionate combination (SFC) or with tiotropium bromide. If optimal management is not achieved on one of these two treatments and patients remain symptomatic or experience an exacerbation, patients have the option to step up to treatment with both drugs, ie, TRIPLE therapy. This paper summarizes the methodology of the study in advance of the publication of the results.

\section{Methods \\ Study design}

This study is a 24-week, multicenter, randomized, parallelgroup study. Initial study treatments will be double-blind and double-dummy. This study will include a switch treatment strategy for those not adequately controlled on randomized medication. The study aims to recruit 400 patients with moderate-to-severe COPD; 200 patients per treatment arm. After a 4-week run-in period, during which patients will remain on their usual treatment for COPD, patients will be randomized to receive SFC (Adoair ${ }^{\mathrm{TM}}$, GlaxoSmithKline KK, Tokyo, Japan) 50/250 $\mu \mathrm{g}$ twice daily delivered via the Diskus ${ }^{\mathrm{TM}}$ (GlaxoSmithKline KK) inhalation device, or tiotropium bromide (Spiriva ${ }^{\mathrm{TM}}$, Boehringer Ingelheim Pharma $\mathrm{GmbH}$ and $\mathrm{Co}, \mathrm{KG}$ Ingelheim, Germany) $18 \mu \mathrm{g}$ delivered once daily via the Handihaler ${ }^{\mathrm{TM}}$ inhalation device (Boehringer Ingelheim Pharma $\mathrm{GmbH}$ and $\mathrm{Co}$ ), for 24 weeks. SFC $50 / 250 \mu \mathrm{g}$ is the licensed dose in Japan. Within the doubledummy study design (Figure 1), patients in both groups also receive placebo medication via a matched-placebo inhalation device. Subjects treated with TRIPLE therapy will receive open-label medication. During the run-in, regular treatment with any COPD medications is allowed, except for systemic corticosteroids and antibiotics used for COPD exacerbation. The use of other COPD medication such as antitussives, expectorants, and mucolytics is permitted throughout the study at the investigator's discretion. Salbutamol for use on an as required basis for relief medication, short-term oxygen therapy for treatment of COPD exacerbation, and short-term courses of systemic steroids and/or antibiotics for a COPD exacerbation or adverse event (AE) will also be allowed. No ICS, LABAs, leukotriene antagonists, phosphodiesterase (PDE)-4 inhibitors, anticholinergics, and regular or longterm oxygen therapy ( $\geq 12$ hours oxygen use per day) will be 


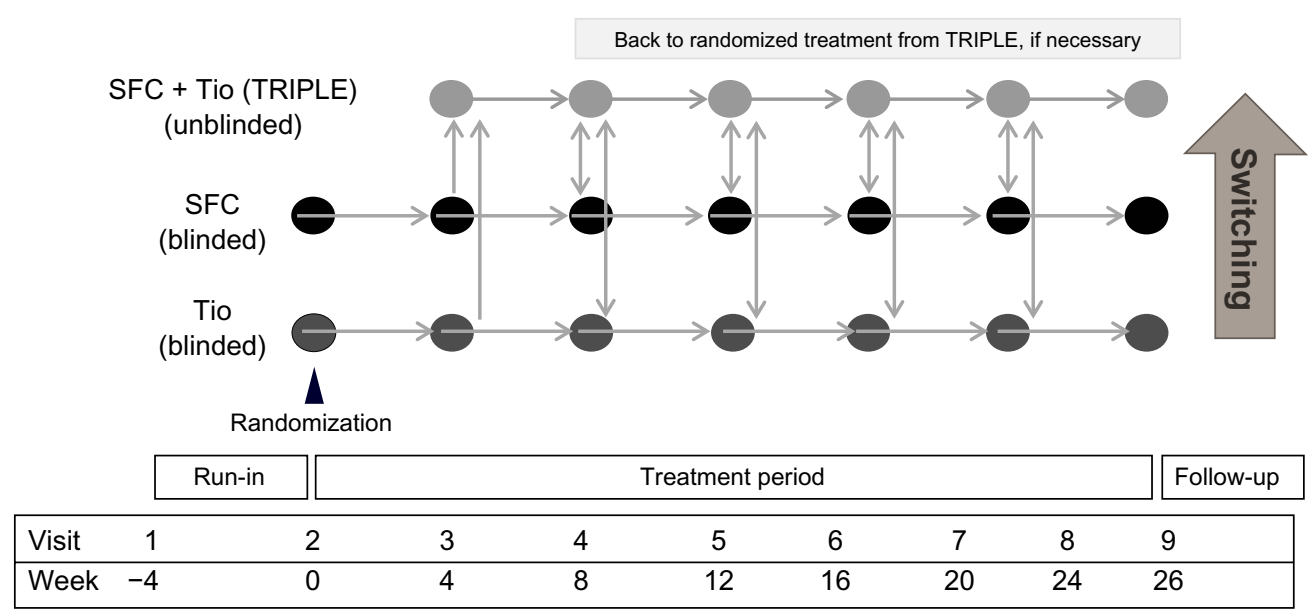

Figure I Study design.

Abbreviations: SFC, salmeterol/fluticasone propionate; Tio, tiotropium bromide; TRIPLE, SFC twice daily and Tio once daily.

allowed, except for randomized study treatment. If a moderate or severe exacerbation occurs more than once or the CAT score is increased by more than four points after switching to TRIPLE therapy, regular use of COPD medications other than systemic steroids, ICS, $\beta_{2}$-agonists, and anticholinergic agents is permitted.

\section{Study protocol}

Post-randomization, patients will be reviewed every 4 weeks to assess their symptom level and the occurrence of any exacerbations (Figure 1). This will be done using the CAT, and asking whether there have been any noticeable changes since the last visit and whether an exacerbation has occurred. $\mathrm{FEV}_{1}$ and forced vital capacity (FVC) will also be measured at each visit.

Based on the CAT score and presence or absence of exacerbations, at each visit the investigator will ask the subject

Table I Guidance for COPD control on randomized therapy

\begin{tabular}{|c|c|}
\hline CAT/exacerbation & Guidance \\
\hline CAT $<10$ & $\begin{array}{l}\text { "Your score suggests you are doing } \\
\text { well. Are you satisfied with your current } \\
\text { treatment?" }\end{array}$ \\
\hline $10 \leq \mathrm{CAT} \leq 15$ & $\begin{array}{l}\text { "Your score suggests your chest } \\
\text { trouble is causing you some problems } \\
\text { because }[. . .] \text {. Are you satisfied with your } \\
\text { current treatment?" }\end{array}$ \\
\hline CAT $>15$ & $\begin{array}{l}\text { "Your score clearly suggests your chest } \\
\text { problem is having a big effect on you. We } \\
\text { have an option to increase treatment. Do } \\
\text { you want to try it?" }\end{array}$ \\
\hline Moderate or severe & Switch to TRIPLE therapy \\
\hline COPD exacerbation & \\
\hline
\end{tabular}

Abbreviations: CAT, COPD Assessment Test; SFC, salmeterol/fluticasone propionate; Tio, tiotropium bromide; TRIPLE, SFC twice daily and Tio once daily; COPD, chronic obstructive pulmonary disease. questions in accordance with the guidance for optimization of COPD treatment (Table 1). Randomized treatment will be switched to TRIPLE therapy if necessary, having taken the subject's answers and his/her medical course into consideration and having obtained their agreement. The necessity of switching treatments to TRIPLE therapy should be assessed at unscheduled visits, if any, as well as at scheduled visits.

At the first scheduled visit after switching to TRIPLE therapy, TRIPLE therapy should be continued to the next scheduled visit even if there is no improvement in symptoms, since benefits may take more than 1 month to develop.

At the second scheduled visit after switching to TRIPLE therapy, if COPD symptoms are not improved, or if the subject is unsatisfied with the study treatment in spite of switching to TRIPLE therapy, or they have developed side effects due to the added treatment, the investigator should consider changing treatments back to the double-blinded randomized medication. Following a switch back from TRIPLE to the randomized treatment, the physician can return to TRIPLE, if the patient's COPD symptoms justify it or if the subject is unsatisfied with the randomized treatment.

In cases when patients are changed to TRIPLE because of a moderate or severe COPD exacerbation, TRIPLE therapy should be continued throughout the course of the study. The investigator should consider adding other COPD medications or suitable treatment if an exacerbation has occurred more than once during the study or the CAT score has increased more than four points despite switching to TRIPLE therapy.

\section{Patient participation}

Patients will be aged 40-80 years with an established clinical history of COPD as defined by the GOLD 
guidelines, ${ }^{4}$ with a current or former smoking history of $\geq 10$ pack years, a post-bronchodilator $\mathrm{FEV}_{1}$ of $\geq 30 \%$ to $\leq 80 \%$ of the predicted normal value, a post-bronchodilator $\mathrm{FEV}_{1} / \mathrm{FVC}$ ratio of $<70 \%$, and a grade of $\geq 1$ on the mMRC scale. ${ }^{18}$ Exclusion criteria for entry to the study include a medical diagnosis of predominant asthma or a respiratory disorder other than COPD that might interfere with the study, lung transplantation and/or lung volume reduction, and a requirement for regular or long-term oxygen therapy. Patients on long-term oxygen therapy would be unlikely to be able to complete the study, with long-term oxygen therapy defined as $\geq 12$ hours of oxygen a day. Other exclusion criteria include a plan to start or change to a pulmonary rehabilitation program during the study period, regular treatment with oral, parenteral, or depot corticosteroids, a chest X-ray indicating a diagnosis other than COPD, or any other serious, uncontrolled disease likely to interfere with the study. Patients who have received any other investigational drug within 4 weeks of the study are also excluded from the study. As with other anticholinergic drugs, patients with a medical diagnosis of narrow-angle glaucoma, prostatic hyperplasia, or bladder neck obstruction should only be entered at the investigator's discretion.

Randomization is remote and concealed from centers and uses a 1:1 allocation ratio. The primary population for analysis is the modified intent-to-treat (m-ITT) population, which will include all patients randomized to treatment who have received at least one dose of trial medication.

The study is being conducted in accordance with the International Conference on Harmonisation Good Clinical Practice and the 2008 version of the Declaration of Helsinki. All patients must provide a written informed consent form prior to participation. The ethics and review boards of all participating institutions have approved the protocol (GSK study code: SCO116717; NCT01762800). ${ }^{19}$

\section{Efficacy assessments}

The primary efficacy endpoint is the proportion of patients who are able to remain on the randomized therapy. Secondary and other efficacy endpoints are listed in Table 2.

\section{Exacerbations}

A COPD exacerbation is defined as a sustained worsening of COPD symptoms beyond normal day-to-day variations. The investigator will record all COPD exacerbations throughout the study period in accordance with the classification of exacerbation severity.
Table 2 Secondary endpoints

Proportion of patients who switched to TRIPLE therapy
Proportion of patients controlled by TRIPLE therapy
Proportion of patients controlled by randomized therapy plus TRIPLE
therapy
Time to switching to TRIPLE therapy
Time to first exacerbation
Proportion of exacerbation confirmed by EXACT
Proportion of exacerbations detected by EXACT not diagnosed
CAT score change
Change in FEV
Use of relief medication
Proportion of patients who decreased treatment from TRIPLE therapy
Proportion of patients who required additional treatment to TRIPLE
therapy
Proportion of patients who dropped out
Patient's judgment of treatment efficacy
Physician's judgment of treatment efficacy

Abbreviations: CAT, COPD Assessment Test; EXACT, EXAcerbations of Chronic Pulmonary Disease Tool; FEV , forced expiratory volume in I second; SFC, salmeterol/fluticasone propionate; TRIPLE, SFC twice daily and tiotropium once daily; COPD, chronic obstructive pulmonary disease.

- An exacerbation will be classified as a mild exacerbation if treated with relief medication only.

- An exacerbation will be classified as a moderate exacerbation if treated with systemic corticosteroids and/ or antibiotics.

- An exacerbation will be classified severe exacerbation if hospitalization is required for treatment of the exacerbation.

In this study, both reported and unreported COPD exacerbations will be assessed using the EXAcerbations of Chronic Pulmonary Disease Tool (EXACT). ${ }^{20-22}$ Unreported exacerbations will be determined from symptoms recorded by patients on daily record cards. After the screening visit, subjects will record their respiratory symptoms and physical condition every day. Use of relief medication will also be recorded on the daily record cards.

\section{Safety assessment}

Safety will be measured by recording any AEs and COPD exacerbations.

\section{Statistical analysis}

Assuming a $10 \%$ to $60 \%$ switch rate from the initial treatment group, a $15 \%$ difference will be detectable with $86 \%$ to $99 \%$ power by log-rank test using the planned sample size of 400 subjects.

For the primary comparison of interest, the proportion of patients remaining on randomized therapy (=1-TRIPLE switch rate) and time to switch to TRIPLE will be estimated 
by Cox's proportional hazard model. A Kaplan-Meier plot will be prepared.

Descriptive statistics of $\mathrm{FEV}_{1}$ and CAT scores will be presented. AEs will be coded according to the Medical Dictionary for Regulatory Activities (MedDRA) and summarized by body systems and preferred terms. Serious AEs, drug-related AEs, and events leading to withdrawal will also be summarized.

The primary analysis population will be the m-ITT, which will consist of randomized subjects who received at least a single dose of the investigational product.

\section{Discussion}

It is now recognized that the treatment of COPD should involve more than improving lung function since the impact of lung function impairment varies greatly between individual patients. Exacerbations and symptoms also have a significant impact on the health status of these patients. ${ }^{4,23-25}$ As a result, the GOLD 2011 Strategy Document, updated in 2013, has produced a treatment algorithm based on the assessment of symptoms and exacerbations with the aim of enabling more effective treatment of COPD. ${ }^{5,6}$ However, this strategy has not been tested in a clinical trial, and it is also not clear what proportion and what type of patients can be managed on a single therapy and which should receive multiple therapies. This study will attempt to clarify aspects of this and to determine whether this strategy can improve the management of COPD, particularly in a Japanese setting.

This strategy cannot easily be tested in a usual randomized clinical trial design since this does not reflect real clinical practice (eg, withdrawal from a study often occurs when the study treatment to which the patient is randomized in a blinded manner is judged to be insufficiently effective). Moreover, subject withdrawals from randomized clinical trials are usually the sickest patients who, in real clinical practice, are those who need the most help. ${ }^{26} \mathrm{~A}$ further complication of withdrawals in randomized clinical trials is that this risks a significant study bias. ${ }^{27}$ It is known that long-term trials in COPD present difficult problems of design and statistical analysis. ${ }^{27}$

In order to test the GOLD strategy, a new type of study design has therefore been introduced. It starts like a usual type of clinical trial with two randomized, blinded arms of appropriate COPD treatments. At each visit, the investigator then assesses the effectiveness of the treatment by assessing symptoms (checking the CAT score, asking the subject how they feel about their treatment using formal study-designed questions, and checking for exacerbation events). The Japanese version of the CAT has been shown to have high reliability and validity for precise assessment of the health status of Japanese patients with COPD. ${ }^{9}$ It is important to note that lung function measurements are not used as criteria for treatment change in this study. The primary efficacy endpoint is the proportion of patients who are controlled and able to remain on the randomized therapy. At each assessment point, if the treatment is judged to be satisfactory, the patient's treatment remains unchanged. However, if the treatment is not felt to be effective, instead of the patient having to withdraw or continue on the same therapy but under-treated in terms of their symptoms, the investigator can increase the treatment. This process will occur at each visit and, depending on treatment response, there are options in this study design to maintain, increase, or reduce the treatment as appropriate, thus mimicking clinical practice as far as possible.

TRIPLE therapy, which adds an LAMA to ICS/LABA combinations, has been associated with greater improvements in lung function and quality of life and reduced rates of hospitalization compared with anticholinergic therapy alone. ${ }^{13-17}$ While the efficacy and tolerability of TRIPLE therapy have been evaluated in clinical trials, the effect on COPD outcomes of TRIPLE therapy relative to LAMA and SFC alone has not been assessed outside the confines of a controlled clinical trial.

The strengths of this study design are that a level of treatment flexibility is allowed, which better reflects real clinical practice than a randomized clinical trial and this should also ensure that the sicker patients are not lost from the study. This makes it possible to test the hypothesis behind the GOLD 2011 treatment strategy. The principal weakness in the study design is that when treatment is increased, the blinding will be lost. However, there has to be a compromise to achieve the study objectives and this is probably the best solution. An additional limitation is that the only valid options for treatment are an LAMA or combined LABA/ICS therapy, and that the next obvious step is TRIPLE. However, adding an LABA to an LAMA (without an ICS) may be a valid option for patients not controlled with an LAMA alone in terms of symptoms.

If this study design is successful, the methodology can be used for future studies with new therapies and may complement the standard fixed treatment approach of randomized clinical trials for drug registration purposes. The results of this study will also help physicians to understand if TRIPLE therapy is more effective in controlling symptoms and exacerbations in patients with moderate 
to severe COPD when compared with either treatment strategy alone. This study will also help physicians to understand the GOLD recommendation work in Japan when communicating with their patients and with using the available medications.

\section{Acknowledgments}

The authors are grateful to United BioSource Corporation, the developer of the EXAcerbations of Chronic Pulmonary Disease Tool (EXACT). The authors are indebted to the sponsor's project staff for their support and contributions throughout the protocol development. We are grateful to all teams of GSK for their contribution to this study, especially Yu Jincho, Tomoyuki Hayamizu, Hideo Kikkawa, and Kazuyoshi Tenjinbaru for protocol development. The authors thank Diana Jones (Cambrian Clinical Associates Limited) for medical writing and manuscript coordination, which was funded by the study sponsor.

\section{Author contributions}

Professor Tomoko Betsuyaku, Dr Motokazu Kato, Professor Keisaku Fujimoto, Dr Gerry Hagan, Hideki Hitosugi, Mark James, Paul W Jones: COSMOS-J steering committee members, protocol development. Akihiro Kobayashi: statistical contribution for protocol development. All authors were involved in the acquisition and analysis of data, drafting and critical revision of the manuscript, and the final approval of the proof to be published.

\section{Disclosure}

GlaxoSmithKline KK (GSK) is the funding source and is involved in all stages of the study protocol development. GSK also took in charge all costs associated with the development and the publishing of the present manuscript. All authors took final responsibility for submitting for publication.

Paul W Jones discloses that his university has received honoraria and research grants from the commercial entity that sponsored the study. Tomoko Betsuyaku discloses having received honoraria/paid expert testimony and her university having research grants from the commercial entity that sponsored the study. Keisaku Fujimoto discloses receiving consulting fees from GSK for participating on the steering committee of the study. Motokazu Kato discloses having received consulting fees from GSK for participating on the steering committee of the study and having received consultancy fees from other pharmaceutical companies. Gerry Hagan discloses having received a consultancy fee from GSK and reports ownership of GSK stock options.
Hideki Hitosugi, Akihiro Kobayashi, and Mark James are employees of GlaxoSmithKline and report ownership of GSK stock options.

\section{References}

1. The Japanese Respiratory Society. Guidelines for the Diagnosis and Treatment of COPD. 3rd ed. Tokyo: Japanese Respiratory Society; 2009.

2. Lopez AD, Shibuya K, Rao C, et al. Chronic obstructive pulmonary disease: current burden and future projections. Eur Respir J. 2006;27: 397-412.

3. Mathers CD, Loncar D. Projections of global mortality and burden of disease from 2002 to 2030. PLoS Med. 2006;3(11):e442.

4. Global initiative for chronic Obstructive Lung Disease (GOLD) [homepage on the Internet]. 2010. Available from: http://www.goldcopd. org/Guidelines/guideline-2010-gold-report.html. Accessed March 25, 2013.

5. Global initiative for chronic Obstructive Lung Disease (GOLD). 2011; http://www.goldcopd.org/Guidelines/guideline-2011-gold-report.html. Accessed March 25, 2013.

6. Global initiative for chronic Obstructive Lung Disease (GOLD). 2013; http://www.goldcopd.org/Guidelines/guideline-2013-gold-report.html. Accessed March 25, 2013.

7. Jones PW, Harding G, Berry P, Wiklund I, Chen WH, Kline Leidy N. Development and first validation of the COPD Assessment Test. Eur Respir J. 2009;34:648-654.

8. Jones PW, Brusselle G, Dal Negro RW, et al. Properties of the COPD assessment test in a cross-sectional European study. Eur Respir J. 2011;38:29-35.

9. Tsuda T, Suematsu R, Kamohara K, et al. Development of the Japanese version of the COPD Assessment Test. Respir Investig. 2012;50: 34-39.

10. Ichinose $\mathrm{M}$, Aizawa $\mathrm{H}$, Ishizaka $\mathrm{A}$, et al. [Chronic obstructive pulmonary disease (COPD) burden in Japan: confronting COPD Japan survey.] Nihon Kokyuki Gakkai Zasshi. 2007;45(12):927-935. Japanese [with English abstract].

11. Fukuchi Y, Nishimura M, Ichinose M, et al. COPD in Japan: the Nippon COPD Epidemiology study. Respirology. 2004;9:458-465.

12. Nakano Y, Nagai A, Mishima M, Yoshimoto D, Hagan G. [Investigation of COPD management in primary care in Japan using hand-held spirometer.] Kokyu. 2012;31:273-281. Japanese.

13. Aaron SD, Vandemheen KL, Fergusson D, et al; Canadian Thoracic Society/Canadian Respiratory Clinical Research Consortium. Tiotropium in combination with placebo, salmeterol, or fluticasonesalmeterol for treatment of chronic obstructive pulmonary disease: a randomized trial. Ann Intern Med. 2007;146:545-555.

14. Perng DW, Wu CC, Su KC, Lee YC, Perng RP, Tao CW. Additive benefits of tiotropium in COPD patients treated with long-acting beta agonists and corticosteroids. Respirology. 2006;11:598-602.

15. Singh D, Brooks J, Hagan G, Cahn A, O'Connor BJ. Superiority of 'triple' therapy with salmeterol/fluticasone propionate and tiotropium bromide versus individual components in moderate to severe COPD. Thorax. 2008;63:592-598.

16. Welte T, Miravitlles M, Hernandez P, et al. Efficacy and tolerability of budesonide/formoterol added to tiotropium in patients with chronic obstructive pulmonary disease. Am J Respir Crit Care Med. 2009; 180:741-750.

17. Hanania NA, Crater GD, Morris AN, Emmett AH, O'Dell DM, Niewoehner DE. Benefits of adding fluticasone propionate/salmeterol to tiotropium in moderate to severe COPD. Respir Med. 2012;106(1): 91-101.

18. Bestall JC, Paul EA, Garrod R, Garnham R, Jones PW, Wedzicha JA. Usefulness of the Medical Research Council (MRC) dyspnoea scale as a measure of disability in patients with chronic obstructive pulmonary disease. Thorax.1999;54:581-586. 
19. GlaxoSmithKline. Evaluating the Control of COPD Symptoms in Patients Treated With Tiotropium Bromide $18 \mathrm{mcg}$ Once Daily Alone, ADOAIR 50/250mcg Twice Daily Alone or ADOAIR 50/250mcg Plus Tiotropium Bromide $18 \mathrm{mcg}$. In: ClinicalTrials.gov [website on the Internet]. Bethesda, MD: US National Library of Meicine; 2012 [updated July 11, 2013]. Available from: http://www.clinicaltrials.gov/ ct2/show/NCT01762800?term=NCT01762800\&rank=1. NLM identifier: NCT01762800. Accessed August 28, 2013.

20. Leidy NK, Wilcox TK, Jones PW, Roberts L, Powers JH, Sethi S; EXACTPRO Study Group. Standardizing measurement of chronic obstructive pulmonary disease exacerbations: reliability and validity of a patientreported diary. Am J Respir Crit Care Med. 2011;183:323-329.

21. Jones PW, Chen WH, Wilcox TK, Sethi S, Leidy NK; EXACT-PRO Study Group. Characterizing and quantifying the symptomatic features of COPD exacerbations. Chest. 2011;139(6):1388-1394.

22. Leidy NK, Wilcox TK, Jones PW, et al; EXACT-PRO Study Group. Development of the EXAcerbations of Chronic Obstructive Pulmonary Disease Tool (EXACT): a patient-reported outcome measure. Value Health. 2010;13:965-975.
23. Schmier JK, Halpern MT, Higashi MK, Bakst A. The quality of life impact of acute exacerbations of chronic bronchitis (AECB): a literature review. Qual Life Res. 2005;14:329-347.

24. Sin DD, McAlister FA, Man SF, Anthonisen NR. Contemporary management of chronic obstructive pulmonary disease: scientific review. JAMA. 2003;290(17):2301-2312.

25. Bahadori K, FitzGerald JM. Risk factors of hospitalization and readmission of patients with COPD exacerbation: a systematic review. Int J Chron Obstruct Pulmon Dis. 2007;2:241-251.

26. Vestbo J, Anderson JA, Calverley PM, et al. Bias due to withdrawal in long-term randomised trials in COPD: evidence from the TORCH study. Clin Respir J. 2011;5:44-49.

27. Keene ON, Vestbo J, Anderson JA, et al. Methods for therapeutic trials in COPD: lessons from the TORCH trial. Eur Respir J. 2009;34: 1018-1023.

\section{Publish your work in this journal}

The International Journal of COPD is an international, peer-reviewed journal of therapeutics and pharmacology focusing on concise rapid reporting of clinical studies and reviews in COPD. Special focus is given to the pathophysiological processes underlying the disease, intervention programs, patient focused education, and self management protocols.

\section{Dovepress}

This journal is indexed on PubMed Central, MedLine and CAS. The manuscript management system is completely online and includes a very quick and fair peer-review system, which is all easy to use. Visit http://www.dovepress.com/testimonials.php to read real quotes from published authors. 